\title{
Synthesizing tomorrow
}

\author{
Synthetic biology could be seen as a natural development of traditional biotechnology and applied \\ genetics. However, the exuberant culture that it has embraced should ensure it has a very bright future.
}

The manipulation of successive generations of plants and animals to better suit the needs and desires of humans has a long history stretching back at least to the emergence of agriculture 8,000 or more years ago. It could even be argued that all the major changes in human civilizations have been associated with ever more efficient and sophisticated ways of adapting the organisms with which humankind shares the Earth. The latest of these processes goes under the name of synthetic biology and its application to plants is bearing early fruits.

Like all scientific advances synthetic biology is not a wholly new discipline. Rather it has grown out of more traditional fields such as biotechnology and genetics. In an attempt to put an end to essentially semantic debates about what is and what isn't synthetic biology a working group set up by the European Commission recently came up with the following definition:

"SynBio is the application of science, technology and engineering to facilitate and accelerate the design, manufacture and/or modification of genetic materials in living organisms." A broad take on the field for sure, yet including the crucial concept of design. A synthetic biologist should not be tinkering with the genetic makeup of an organism hoping for a helpful result, but be making specific adaptions to achieve predictable results.

Initially, much of the commercial interest in synthetic biology focussed on the production of biofuels. As Craig Venter put it back in 2010: "Designing and building synthetic cells will be the basis of a new industrial revolution. The goal is to replace the entire petrochemical industry." The energy in petroleum is after all only sunlight captured by photosynthesis millions of years ago, why not cut down the waiting time? This remains a goal of companies such as Sapphire Energy of San Diego, California which is using engineered algae to produce 'green crude'; aiming for 5,000 barrels a day from their algae farm in New Mexico by 2018 . However, in the half decade since Venter's dramatic prediction, the price of oil has dropped making the commercial viability of such ventures less secure. The hydrocarbon-based synthetic biology firms are looking to diversify their products to include cosmetics, spices, and even food oils.
It would be wrong to think that synthetic biology is restricted to the engineering of single-celled organisms. Crop plants have been making foodstuffs for millennia so it should be no surprise that some recent successes in the field have been achieved in plants considerably more complex than an alga. Camelina plants engineered to produce omega-3 long-chain polyunsaturated fatty acids are currently undergoing field trials in the UK. If all goes well these will become a source of supplements for the fish farming industry as, somewhat ironically, omega-3 fatty acids, though commonly known as fish oils, are not produced by fish but are acquired from other organisms in the marine food chain. Also in the UK, the Norwich-based start-up Persephone Bio is using tomatoes as a platform for producing complex chemicals such as anthocyanins and flavonols as raw materials for the cosmetics industry.

\section{It could be argued that all} the major changes in human civilizations have been

associated with ever more efficient and sophisticated ways of adapting the organisms with which humankind shares the Earth.

Despite, or perhaps because of, its high-tech nature, synthetic biology has taken much inspiration from computer programming and has developed something of a 'hacker' culture. In a push for 'democratization', considerable effort has been put into creating the equivalent of 'open source' code, with the code not binary and computer readable but nucleic acid based. Almost a decade ago the BioBricks Foundation was set up "to ensure that the engineering of biology is conducted in an open and ethical manner to benefit all people and the planet". Chief among its aims was the enabling of sharing standard biological components, the BioBricks of its name. The concept has taken root and there are now over 35,000 plasmids available from
AddGene, the non-profit organization that manages plasmid sharing for BioBricks and others. Among these, 241 are plant specific including a whole modular cloning tool-kit created by the Sainsbury Laboratory in Norwich, UK.

There is also a playfulness associated with synthetic biology. Glowing Plant - a startup established in 2013 with a Kickstarter campaign that received more than seven times its US $\$ 65,000$ funding target - is engineering bioluminescent plants by introducing luciferin from fireflies. They are currently working on Arabidopsis but claim an ultimate goal of trees that can be used for street lighting and house plants that can double as reading lamps. Another start-up, Revolution Bioengineering, is busy creating a Petunia variety whose circadian clock is used to affect the environment of the anthrocyanins that give the flowers their colour. The result? A flower which changes colour with the time of day.

Possibly the greatest demonstration of synthetic biology's commitment to openness, fun and the future is the International Genetically Engineered Machine (iGEM) competition. This annual contest began in 2004 with five teams of undergraduates and has grown dramatically with 2,300 participants in more than 240 teams competing in 2014. Teams must design and engineer their own biological machine using a collection of standard parts (BioBricks) and components they create themselves.

Many of the iGEM teams choose to work in yeast or bacteria but in 2014 a few flew the flag for the plant kingdom. Two teams worked with Nicotiana benthamiana: one to create 'green canaries', sentinel plants that identify the presence of plant pathogens in a crop, and another to manufacture insect sex pheromones to disrupt the reproduction of herbivorous moths. A third team created a biosensor in the liverwort Marchantia polymorpha, which when triggered produced both a change in the plant's colour and its smell.

It can be difficult to see what could not be achieved by synthetic biology. What the success of the iGEM competition shows is that the next generation of synthetic biologists have more than enough skill, imagination and enthusiasm to create wonders. 